\section{Consensus on diagnosis and empiric antibiotic therapy of febrile neutropenia}

\author{
Nagua Giurici, ${ }^{1}$ Giulio A. Zanazzo² \\ 1Department of Pediatrics, IRCCS Burlo \\ Garofolo, Trieste; 2Department \\ of Oncohematology, IRCCS Burlo \\ Garofolo, Trieste, Italy
}

\begin{abstract}
Controversial issues on the management of empiric therapy and diagnosis of febrile neutropenia (FN) were faced by a Consensus Group of the Italian Association of Pediatric Hematology-Oncology (AIEOP). In this paper we report the suggestions of the consensus process regarding the role of aminoglycosides, glycopeptides and oral antibiotics in empiric therapy of FN, the rules for changing or discontinuing the therapy as well as the timing of the blood cultures.
\end{abstract}

\section{Introduction}

A survey of 34 AIEOP centers was carried out in 2010 requesting local policies, protocols and guidelines relating to the management of febrile neutropenia (FN) in children treated for malignant disease. The results of the survey showed variability across centers in many topics. In order to offer a homogeneous care to children treated for cancer, a consensus process was promoted by AIEOP Supportive Care and Infectious Disease Groups to settle the more frequent questions. This paper will focus on the results regarding empiric antibiotic therapy and bacterial diagnosis.

\section{Methods}

Consensus process developed step by step. First step was definition by members of the AIEOP Supportive Care and Infectious Disease Groups of most controversial issues. Second step was a review of literature done by single members of the Consensus Group. Third step was the discussion of results by the delegates of all AIEOP centers in plenary session. At the end of the plenary meeting AIEOP Consensus Group made a consensus on the controversial issues.

\section{Results}

\section{Role of aminoglycosides in empiric therapy}

Even in children with high-risk FN, aminoglycoside-based combined therapies have not demostrated superiority over monotherapy with imipemenics ${ }^{1}$ or with fourth generation cephalosporines ${ }^{2,3}$ in prospectic randomized trials. Nevertheless many authorities have stressed the potential benefits of antimicrobial synergism of aminoglycosides in treatment of gram-negative bacilli infections ${ }^{4}$ or in treatment of very high risk patients who present with haemodynamic instability or who have evidence of multiresistant infection. ${ }^{5}$

The AIEOP Group agrees with the indications of a recent pediatric review ${ }^{6}$ which recommends aminoglycoside-based therapy in the following conditions: demonstrated $P$. aeruginosa infection, suspected catheter-related infection, evidence of sepsis.

\section{Role of glycopeptides in empiric therapy}

Meta analysis of randomized controlled trials on adult population showed that the addition of anti Gram positive treatment, namely glycopeptides, prior to documentation of a Gram-positive infection, does not improve outcomes, ${ }^{7}$ that glycopeptides should not be routinely used as part of the initial empiric treatment $^{8}$ and can be safely deferred until the documentation of a resistant Gram- positive infection. ${ }^{9}$ There are no randomized controlled trials on pediatric population.

The AIEOP Group agrees with the indication of an up-to-date review ${ }^{6}$ that recommends empiric use of glycopeptides in children with FN in the following conditions: clinical suspicion of a catheter related infection, skin and soft tissue infections in areas in which rates of community acquired MRSA surpass 15\%, bone and joint infections, bacteremia caused by Gram+ cocci before identification and antimicrobial susceptibility determination.

\section{Role of oral antimicrobial therapy}

Controlled trials showed that there was no difference in outcome in oral versus iv outpatient therapy in pediatric low risk FN, suggesting that amoxicillin-clavulanate plus ofloxacin ${ }^{10}$ or amoxicillin-clavulanate in combination with ciprofloxacine ${ }^{11}$ or ciprofloxacine alone ${ }^{12}$ may be the oral regimens of choice.

The AIEOP Consensus Group remarks the need for an accurate definition of low risk patients prior to accepting the suggestion of literature. A subgroup of AIEOP was charged to asses the various scores suggested by literature.
Correspondence: Giulio Zanazzo, Department of Pediatric Oncohematology IRCCS Burlo Garofolo, Trieste, Italy.

Tel. +39.040.3785.497 - Fax: +39.040 .3785 .494 .

E-mail: zanazzo@burlo.trieste.it

Key words: neutropenia, antibiotic therapy.

Received for publication: 16 November 2010. Accepted for publication: 14 December 2010.

This work is licensed under a Creative Commons Attribution 3.0 License (by-nc 3.0).

(c) Copyright N. Giurici and G.A. Zanazzo, 2011

Licensee PAGEPress, Italy

Pediatric Reports 2011; 3:e4

doi:10.4081/pr.2011.e4

\section{Changes in empiric therapy}

Literature on adult population suggests an assessment after 48 hours of empiric therapy. In low risk apyretic and no longer neutropenic patients a switch to oral antibiotics could be considered, while in high risk apyretic and no longer neutropenic patients aminoglycosides could be discontinued. Patients who are still pyrexial have to continue with initial therapy if clinically stable, while if clinically unstable require a modification of the antibiotic regimen (shift to carbapemen, addition of gycopeptide). ${ }^{13}$ Given the lack of specific guidelines for the pediatric population the AIEOP Consensus Group agrees with these suggestions.

\section{When to discontinue empiric therapy}

Literature suggests that antibacterials can be discontinued when neutrophil count is $>500 / \mathrm{mmc}$, the patient is asymptomatic, has been afebrile for $48 \mathrm{~h}$ and blood cultures are negative. If neutrophil count remains $<500 /$ mmc and the patient is asymptomatic antibacterials can be discontinued after 7 days of apyrexia. ${ }^{13}$ AIEOP Consensus Group agrees with these suggestions.

\section{Utility of peripheral blood cultures}

The utility of peripheral blood cultures in febrile neutropenic children with central venous catheters is controversial. In the large majority of AIEOP Centres this practice is actually neglected.

Even though a recent paper ${ }^{14}$ supports continuation of routine peripheral cultures, the apparent advantage could be explained with a larger blood volume collection. In fact low level bacteremia is common in pediatric population, requiring the culturing of up to 4 or $4.5 \%$ of a patient's total blood volume for the reliable detection of pathogens. ${ }^{15,16}$ AIEOP Consensus Group concludes that peripheral blood cultures are not necessary and exhorts to collect an adequate blood volume. 


\section{Timing of blood cultures}

Recent literature points out that the likelihood of documenting bloodstream infections was not enhanced by collecting blood at the time of temperature spikes, ${ }^{17}$ that surveillance blood cultures could detect causative microorganisms in more than one-third of blood stream infections prior to the onset of clinical manifestations ${ }^{18}$ and that blood cultures with an adequate blood volume were more likely than those with an inadequate blood volume to yield positive blood culture results. ${ }^{16}$

In case of FN, AIEOP Consensus Group advises to collect at least two sets of blood cultures (for aerobe and anaerobe germs) at a close interval (15') independently from temperature spikes immediately prior to the start of the empiric therapy.

The AIEOP Consensus Group also exhorts to collect a total blood volume adequate to the weigh of patients ( $6 \mathrm{~mL}$ from 2 up to $12 \mathrm{~kg} ; 20$ $\mathrm{ml}$ from 12 up to $36 \mathrm{~kg}$; $40 \mathrm{~mL}$ up to $36 \mathrm{~kg}$ ).

\section{References}

1. Agaoglu L, Devecioglu 0, Anak S, et al. Cost effectiveness of cefepime+netilmicin or ceftazidime+amikacin or meropenem monotherapy in febrile neutropenic children with malignancy in Tukey. J Chemother 2001;13:281-7.

2. Corapcioglu F, Sarper N. Cefepime versus ceftazidime+amikacin as empirical therapy for febrile neutropenia inc hildren with cancer: a prospective randomized trial of the treatment efficacy and cost. J Pediatr
Hematol Oncol 2005;22:59-70.

3. Pereira CA, Petrilli AS, Carlesse FA, et al. Cefepime monotherapy is as effective as cetriaxone plus amikacin in pediatric patient with caner and high-risk febrile neutropenia in a randomized comparison. J Microbiol Immunol Infect 2009;42:141-7.

4. Klastersky J. Why empirical therapy? J Antimicrob Chemother 2009;63:i14-15.

5. Pizzo PA. Where do we go from here? J Antimicrob Chemother 2009;63:i16-17.

6. Santolaya ME Supportive care in children. Curr Opin Oncol 2010;22:323-9.

7. Paul M, Borok S, Fraser A, et al. Additional anti-Gram-positive antibiotic treatment for febrile neutropenic cancer patients. Cochrane Database Syst Rev 2005;CD00 3914.

8. Vardakas K, Samonis G, Chrysanthopoulou SA, et al. Role of glycopeptides as part of initial treatment of febrile neutropenic patients: a meta analysis of randomized controlled trials. Lancet Infect Dis 2005;5: 431-9.

9. Paul M, Borok S, Fraser A, et al. Empirical antibiotics against Gram-positive infections for febrile neutropenia: systematic review and meta-analysis of randomized controlled trials. J Antimicrob Chemother 2005;55:436-44.

10. Gupta A, Swaroop C, Agarwala S, et al. Randomized control trial comparing oral moxicillin-clavulanate and ofloxacin with intravenous ceftriaxone and amikacina as outpatient therapy in peiatric low-risk febrile neutropenia. J Pediatr Hematol Oncol 2009;31:635-641.

11. Cagol AR, Galvao de Castro CJ, et al. Oral vs intravenous empirical antimicrobial therapy in febrile neutropenic patients receiving childhood cancer chemotherapy. J Pediatr (Rio J) 2009;85:531-5.

12. Petrilli AS, Dantas LS, Campos MC, et al. Oral ciprofloxacin vs intravenous ceftriaxone administered in an outpatient setting for fever and neutropenia in low-risk pediatric oncology patients: randomized prospective trial. Med Pediatr Oncol 2000; 34:87-91.

13. de Naurois J, Novitzky-Basso I, Gill MJ, et al. Management of febrile neutropenia: ESMO Clinical Practice Guidelines. Ann of Oncol 2010;21:v252-6.

14. Scheinemann K, Ethier MC, Dupuis LL, et al. Utility of peripheral blood cultures in bacteremic pediatric cancer patients with a central lin. Support Care Cancer 2010; 18:913-9.

15. Kellogg J, Manzella J, Bankert D. Frequency of low level bacteremia in children from birth to fifteen years of age. J Clin Microbiol. 2000;38 :2181-5.

16. Connell TG, Rele M, Cowley D, et al. How reliable is a negative blood culture result? Volume of blood submitted for culture in routine practice in a children's hospital. Pediatric 2007;119:891-6.

17. Riedel S, Bourbeau P, Swartz B, et al. Timing of specimen collection for blood cultures from febrile patients with Bacteremia. J Clin Microbiol 2008;46: 1381-5.

18. Penack 0, Rempf P, Eisenblätter $\mathrm{M}$, et al. Bloodstream infections in neutropenic patients: early detection of pathogen and directed antimicrobial therapy due to surveillance blood cultures. Ann Oncol 2007; 18:1870-4. 